\title{
Localized pinning states in closed containers: Homoclinic snaking without bistability
}

\author{
Isabel Mercader, Oriol Batiste, and Arantxa Alonso \\ Departament de Física Aplicada, Universitat Politècnica de Catalunya, 08034 Barcelona, Spain \\ Edgar Knobloch \\ Department of Physics, University of California, Berkeley, California 94720, USA \\ (Received 8 September 2008; revised manuscript received 25 June 2009; published 20 August 2009)
}

\begin{abstract}
Binary mixtures with a negative separation ratio are known to exhibit time-independent spatially localized convection when heated from below. Numerical continuation of such states in a closed two-dimensional container with experimental boundary conditions and parameter values reveals the presence of a pinning region in Rayleigh number with multiple stable localized states but no bistability between the conduction state and an independent container-filling state. An explanation for this unusual behavior is offered.
\end{abstract}

DOI: 10.1103/PhysRevE.80.025201

PACS number(s): 47.54.-r, 47.20.Bp, 47.20.Ky

Many physical systems exhibit spatially localized structures or dissipative solitons. Examples arise in nonlinear optics [1], buckling of slender structures [2], and reactiondiffusion systems [3]. Spatially localized oscillations or oscillons are time-dependent structures of this type [4]. Similar structures are found in fluid flows, as indicated by the recent discovery of convectons in binary fluid convection [5-7] and related systems [8-10]. A binary mixture with negative separation ratio heated from below develops a stabilizing concentration gradient via the (anomalous) Soret effect resulting in the presence of subcritical steady convection (Fig. 1). This subcritical regime favors the presence of convectons. These come in two types, even and odd under reflection in a vertical plane through their center, and are located in the so-called pinning region [11]. In this interval of Rayleigh numbers multiple stable convectons, of different lengths and either parity, are present. In horizontally unbounded domains these localized structures appear simultaneously with the (subcritical) primary branch of spatially periodic steady convection. The resulting convectons are spatially extended at small amplitude but become strongly localized when followed numerically to larger amplitude by decreasing the Rayleigh number. Once their amplitude and length is comparable to the amplitude and wavelength of steady spatially periodic convection both convecton branches enter the pinning region and begin to snake back and forth across it (Fig. 1) as the convectons grow in length by nucleating additional convection rolls at both ends. This process continues until the length of the convecton becomes comparable to the available spatial domain when the convecton branches turn over toward the saddle node of the periodic branch and leave the pinning region $[8,10,12]$.

The conventional explanation of this behavior uses the presence of bistability between the conduction and spatially periodic convecting states together with spatial reversibility and relies on the similarity between the observed behavior (Fig. 1) and the corresponding behavior of the SwiftHohenberg equation (SHE) on the real line $[13,14]$. The SHE is variational and so permits a comparison between the energy of the trivial (conduction) state and the energy of the spatially periodic state. When these energies are equal a front from the former to the latter (and vice versa) will be stationary. Thus at this parameter value, $R=R_{M}$, say, multiple local- ized structures embedded in a background filled with the trivial state are possible. When $R<R_{M}$ the energy of the trivial state is lower than that of the structured state but the anticipated front motion is thwarted by the pinning of the fronts to the structure between them [11]. Instead the localized state is compressed but remains stationary. In contrast, when $R>R_{M}$ the state is dilated but remains stationary until the energy difference between the two states is made so large that the fronts unpin, allowing the structured state to invade the whole domain [13]. Qualitatively similar behavior characterizes nonvariational systems as well $[1,3,6,8]$. Indeed, stable convectons are found even when the conduction state is unstable to growing oscillations provided that this instability is convective and not absolute [6].

In this Rapid Communication we describe the changes in snaking behavior due to the presence of lateral boundaries. We show that in a closed container snaking can occur even in the absence of bistability between a trivial and an independent structured state and use our understanding of this be-

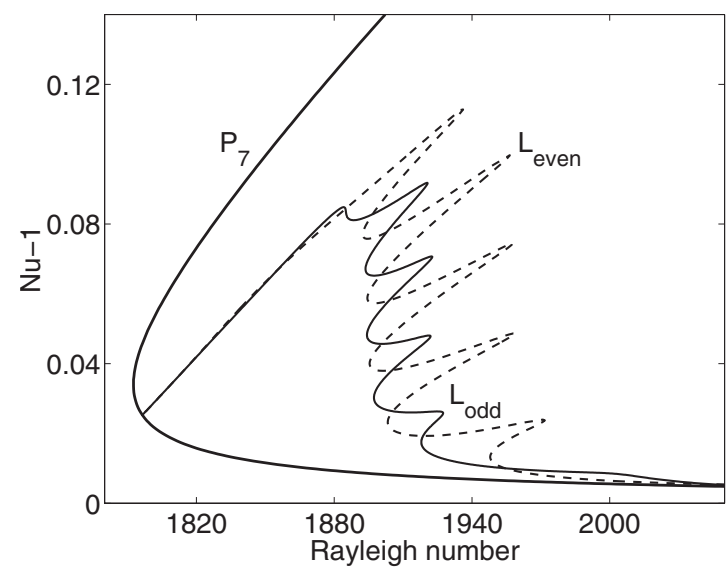

FIG. 1. The branches of stationary spatially periodic $\left(P_{7}\right)$ and stationary spatially localized states of even $\left(L_{\text {even }}\right.$, dashed line $)$ and odd $\left(L_{\text {odd }}\right.$, solid line) parity in a $\Gamma=14$ periodic domain showing the normalized convective Nusselt number $\mathrm{Nu}-1 \equiv \Gamma^{-1} \int_{-\Gamma / 2}^{\Gamma / 2} \partial_{z} \theta(x, z$ $=1) d x$ as a function of the Rayleigh number $R$. The pinning region of the odd convectons is narrower than that of the even convectons. Both convecton branches terminate together on $P_{7}$. Only timeindependent states are shown. 

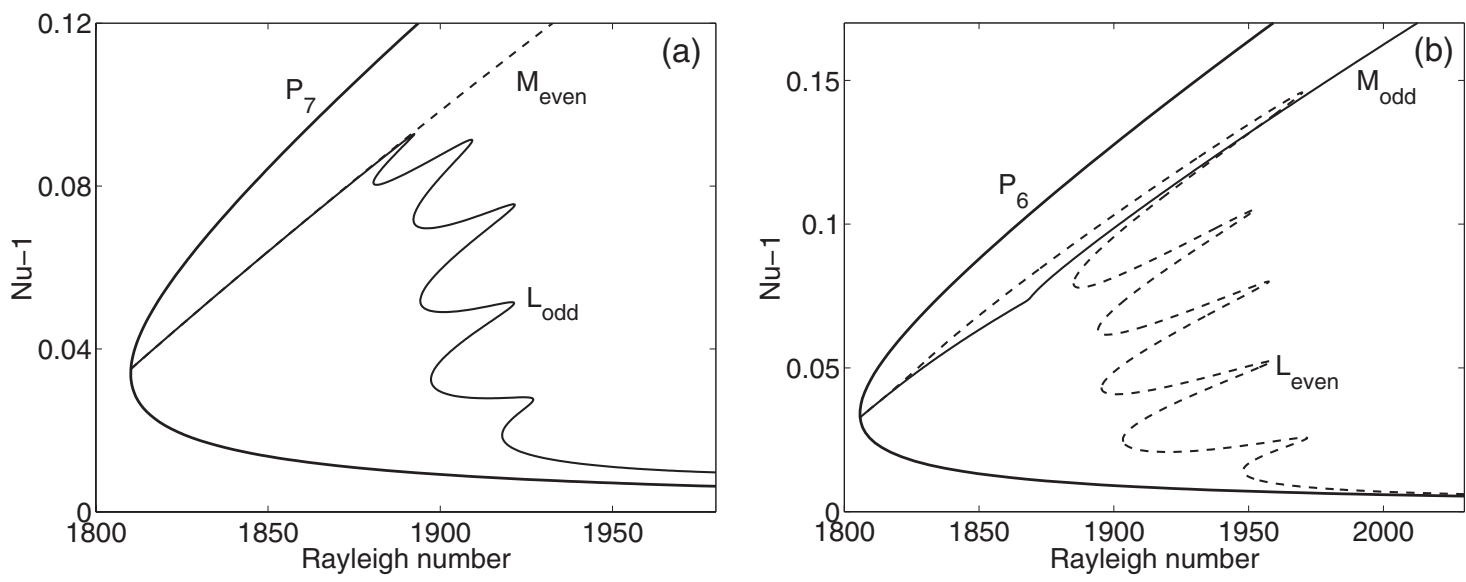

FIG. 2. The branches of spatially periodic $\left(P_{7}\right.$, black line) and spatially localized states of even $\left(L_{\text {even }}\right)$ and odd $\left(L_{\text {odd }}\right)$ parity in a $\Gamma$ $=13$ periodic domain showing the convective Nusselt number $\mathrm{Nu}-1$ as a function of the Rayleigh number $R$. (a) The odd convectons $\left(L_{\text {odd }}\right)$ terminate on $P_{7}$ together with a monotonic branch of even mixed modes $\left(M_{\text {even }}\right)$. (b) The even convectons $\left(L_{\text {even }}\right)$ terminate on $P_{6}$ together with a monotonic branch of odd mixed modes $\left(M_{\text {odd }}\right)$.

havior to explain why in spatially periodic domains the pinning regions for even and odd convectons do not coincide, in contrast to the corresponding situation in the SHE [14].

Binary fluid convection is described by the following equations [6]:

$$
\begin{gathered}
\mathbf{u}_{t}+(\mathbf{u} \cdot \nabla) \mathbf{u}=-\nabla P+\sigma R[(1+S) \theta-S \eta] \hat{\mathbf{z}}+\sigma \nabla^{2} \mathbf{u}, \\
\theta_{t}+(\mathbf{u} \cdot \nabla) \theta=w+\nabla^{2} \theta, \\
\eta_{t}+(\mathbf{u} \cdot \nabla) \eta=\tau \nabla^{2} \eta+\nabla^{2} \theta,
\end{gathered}
$$

together with the incompressibility condition $\nabla \cdot \mathbf{u}=0$. Here $\mathbf{u} \equiv(u, w)$ is the velocity field in $(x, z)$ coordinates, $P$ is the pressure, and $\theta$ denotes the departure of the temperature from its conduction profile, in units of the imposed temperature difference $\Delta T$. The variable $\eta$ is defined such that its gradient represents the dimensionless mass flux. Thus $\eta$ $\equiv \theta-\Sigma$, where $C \equiv 1-z+\Sigma$ is the concentration of the heavier component in units of the concentration difference that develops across the layer via the Soret effect in response to the imposed $\Delta T$. The system is specified by four dimensionless parameters: the Rayleigh number $R$ that provides a dimensionless measure of $\Delta T$, the separation ratio $S$ that measures the concentration contribution to the buoyancy force due to the Soret effect, and the Prandtl and Lewis numbers $\sigma, \tau$, in addition to the aspect ratio $\Gamma$. The physically appropriate boundary conditions are $\mathbf{u}=\theta=\eta_{z}=0$ on $z=0,1$, with either periodic boundary conditions (PBCs) in the hori-

$\mathrm{R}=1982$

(a)

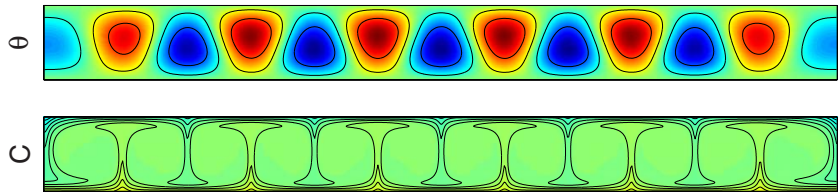

zontal with period $\Gamma$, or the closed container boundary conditions (CCBCs) $\mathbf{u}=\theta=\eta_{x}=0$ on $x= \pm \Gamma / 2$. Throughout we use the (experimental) parameter values $\sigma=7, \tau=0.01$, and $S=-0.1$.

Figure 1 shows the result of numerical branch following in a periodic domain of length $\Gamma=14$. The branches of even and odd convectons (labeled $L_{\text {even }}$ and $L_{\text {odd }}$, respectively) are shown in dashed and solid lines, respectively. Both branches terminate together on the branch $P_{7}$ of stationary periodic states with $n=7$ pairs of rolls, just below the saddle node on the latter, but the pinning region for the odd convectons is noticeably narrower than the corresponding region for even convectons. In contrast, for $\Gamma=13$ the odd branch continues to terminate on $P_{7}$ [Fig. 2(a)], while the even branch terminates on $P_{6}$ [Fig. 2(b)]. This possibility, predicted recently in the context of the SHE $[12,15]$, has also been seen in natural doubly diffusive convection [12]. Since the termination points of $L_{\text {even }}$ and $L_{\text {odd }}$ are always points of weak spatial resonance [12] a second solution branch necessarily terminates at the same locations. These branches correspond to opposite-parity mixed modes [Fig. 3(a)] and extend monotonically to large amplitudes without undergoing snaking [12]. Despite terminating on a different branch the pinning region for odd convectons remains substantially narrower than that for even convectons.

Figure 4 shows the corresponding results with $\mathrm{CCBC}$ and $\Gamma=14$. The pinning regions of even and odd convectons are now essentially identical. Moreover, since periodic states no longer exist both convecton branches emerge from the pin-

FIG. 3. (Color online) (a) The mixed mode $M_{\text {even }}$ in a $\Gamma=14$ domain with PBC, and (b) the corresponding state with CCBC, both at $R=1982$. The latter takes the place of the spatially periodic state $P_{n}$. In each figure the top panel shows contours of constant temperature perturbation $\theta$, while the bottom panel shows contours of constant concentration $C$. 


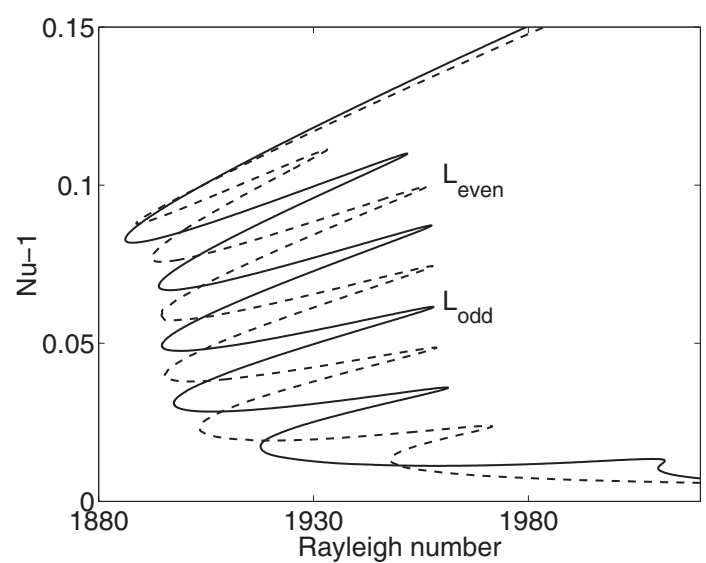

FIG. 4. The corresponding results with $\mathrm{CCBC}$ and $\Gamma=14$. The convecton branches emerge from the pinning region toward the right and there is no branch analogous to $P_{7}$ : at large amplitude the convecton branches resemble the mixed mode states shown in Fig. 2. The width of the pinning region for the even and odd convectons is now the same.

ning region toward large $R$ and gradually turn into (stable) states resembling the mixed modes present with PBC [Fig. 3(b)] albeit with an extra pair of weaker rolls that are responsible for the difference in the homogenized concentration within these states. Thus in this case no separate branch of domain-filling states is present and the bistability between the conduction state and a structured state in Fig. 1 is absent. We conjecture that this type of behavior is always present in systems with non-Neumann boundary conditions.

In Fig. 5 we superpose the branches of even convectons computed with periodic ( $L_{\text {even }}^{\mathrm{PBC}}$, dashed line) and closed container boundary conditions $\left(L_{\text {even }}^{\mathrm{CC}}\right.$, solid line). Within the pinning region the two branches coincide precisely and only begin to differ once they emerge from this region. In contrast, the odd convecton branches differ substantially throughout.
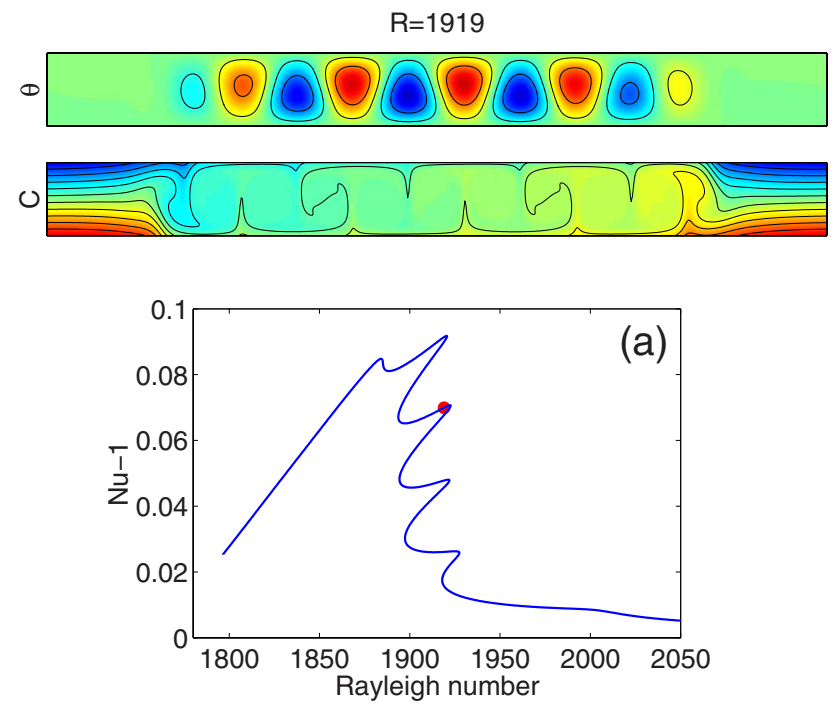

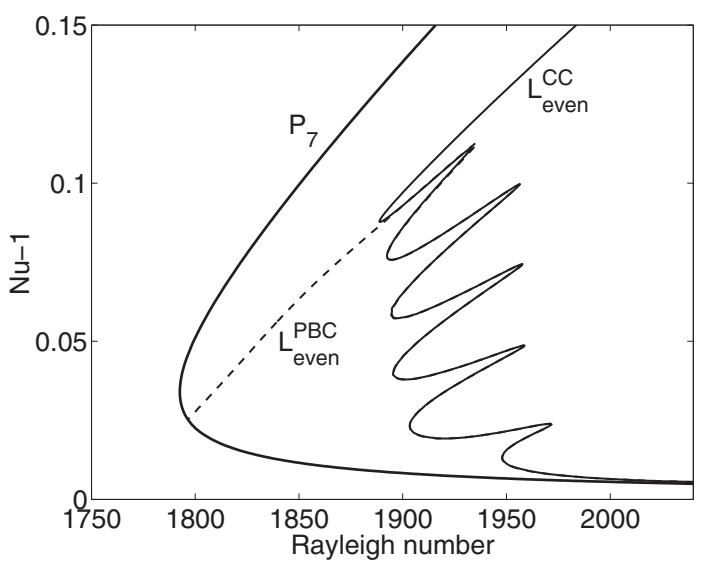

FIG. 5. Superposition of even convecton branches computed in a $\Gamma=14$ domain with $\operatorname{PBC}\left(L_{\text {even }}^{\mathrm{PBC}}\right)$ and $\operatorname{CCBC}\left(L_{\text {even }}^{\mathrm{CC}}\right)$. The $P_{7}$ branch is also shown. Within the pinning region the two branches are identical.

To understand the reason for this behavior we show in Fig. 6 the profiles of the odd convectons in the two cases. Figure 6(a) shows that an odd-parity convecton with $\mathrm{PBC}$ is distinctly asymmetrical, entraining the lighter component from the top on the left and heavier component from the bottom on the right. Such a convecton therefore acts as a pump, pumping concentration from right to left, a fact that is responsible for the slanted isoconcentration contours in the void region outside [6]. In contrast, with CCBC [Fig. 6(b)] the concentration within the convecton is homogeneous, but the concentration contours on the right are substantially depressed in comparison with those on the left. Evidently the state shown in Fig. 6(b) is embedded in an inhomogeneous background state defined by the requirement that in steady state no net concentration (and heat) is transported horizontally.
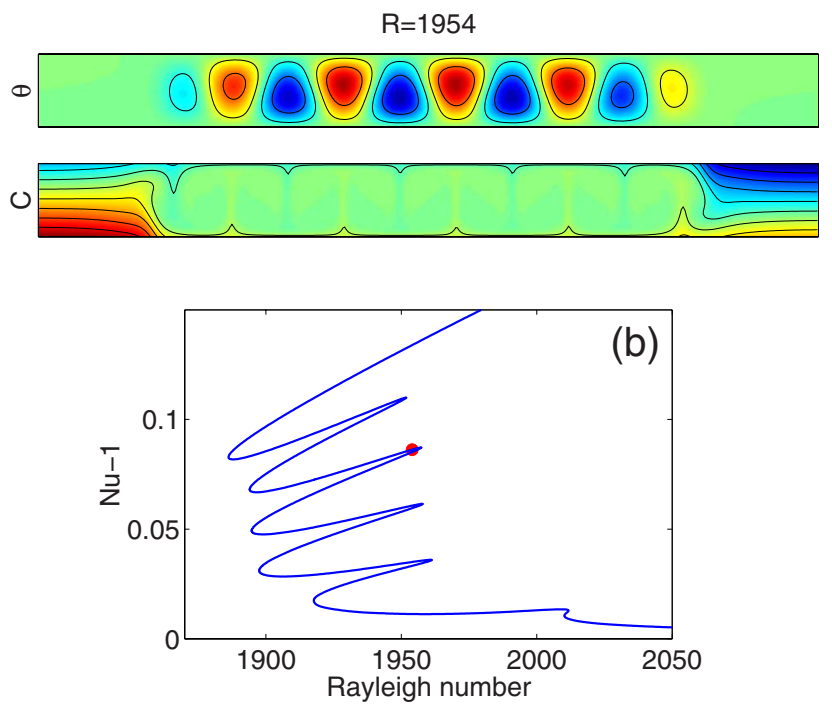

FIG. 6. (Color online) Odd convecton profiles in a $\Gamma=14$ domain with (a) PBC $(R=1919$ and $\mathrm{Nu}-1=0.070)$, and (b) CCBC $(R=1954$ and $\mathrm{Nu}-1=0.086)$. In each figure the top panel shows contours of constant temperature perturbation $\theta$, the middle panel shows contours of constant concentration $C$, while the bottom panel shows the location of the state in the pinning region (solid dots). The state in (a) is markedly asymmetric, in contrast to the state in (b). 

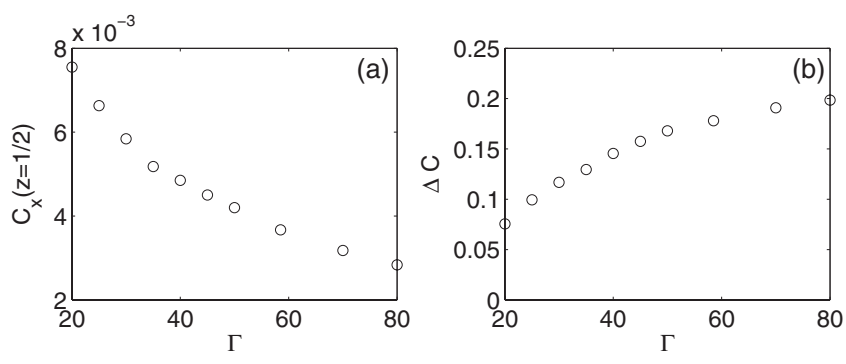

FIG. 7. (a) Background concentration gradient and (b) associated concentration jump $\Delta C$ at midheight across the convectons, both as functions of the domain period $\Gamma$.

Both solutions are invariant under the operation $(x, z)$ $\rightarrow\left(2 x_{0}-x, 1-z\right),(\mathbf{u}, \theta, \Sigma) \rightarrow-(\mathbf{u}, \theta, \Sigma)$, i.e., a reflection in a vertical plane through a suitably chosen origin $x=x_{0}$ followed by a reflection in the layer midplane $z=1 / 2$. Thus in steady state all (vertically averaged) horizontal fluxes necessarily vanish. As shown in Fig. 7(a) the background concentration gradient $C_{x}(x, z=1 / 2)$ decreases with the spatial period $\Gamma$ as $|\Delta C| /(\Gamma-\ell)$, where $\ell$ is the convecton length and $\Delta C$ denotes the midplane concentration difference between the left and right fronts bounding the convecton. Thus the mean concentration gradient within the convecton [Fig. 6(a)] is given by $|\Delta C| / \ell$. Figure 7 (b) reveals that for moderate values of $\Gamma$ the quantity $|\Delta C|$ increases with $\Gamma$ although it must saturate for larger $\Gamma$.

In contrast, in a closed container, constant, albeit different, concentrations are found on either side of the convecton, which is now located in the center of the domain. Solutions of this type are found on a periodic domain of period $2 \Gamma$ whenever two (equidistant) odd-parity states face each other, forming an even-parity state on the larger domain. In contrast, solutions of the first type can also be found in domains of period $2 \Gamma$ but only if the convectons face in the same direction, forming an odd-parity two-pulse state. Thus the closed container odd convectons may be thought of as a part of an even-parity two-pulse state on a larger domain, explaining why states of this type, sufficiently far apart, have the same pinning region as individual even-parity convectons on a periodic domain with period $\Gamma$. On the other hand the odd-parity states in such a domain cannot be continued into a solution of the closed container problem and so remain distinct with distinct finite $\Gamma$ behavior.

The dramatic change from the bifurcation diagram in Fig. 1 to that in Fig. 4 is a consequence of changing the boundary conditions from $\mathrm{PBC}$ to $\mathrm{CCBC}$ and is reproduced by the SHE with non-Neumann boundary conditions [16]. In both systems the behavior observed in Fig. 4 arises as a consequence of the destruction of the spatially periodic state by the boundary conditions appropriate for closed containers. In both cases the remaining mixed modes take over the role of the large amplitude periodic state, and the snaking branches gradually turn into the mixed modes with increasing amplitude. This behavior, referred to here as snaking without bistability, is robust with respect to changes in $\Gamma$.

This work was supported in part by the National Science Foundation under Grant No. DMS-0605238, DGICYT under Grant No. FIS2006-08954, and by AGAUR under Grant No. 2005SGR-0024. We are grateful to A. Bergeon, J. Burke and S. M. Houghton for discussions.
[1] A. G. Vladimirov, J. M. McSloy, D. V. Skryabin, and W. J. Firth, Phys. Rev. E 65, 046606 (2002); J. M. McSloy, W. J. Firth, G. K. Harkness, and G. L. Oppo, ibid. 66, 046606 (2002).

[2] G. W. Hunt et al., Nonlinear Dyn. 21, 3 (2000).

[3] K. J. Lee et al., Nature (London) 369, 215 (1994); V. K. Vanag and I. R. Epstein, Chaos 17, 037110 (2007).

[4] P. B. Umbanhowar, F. Melo, and H. L. Swinney, Nature (London) 382, 793 (1996).

[5] O. Batiste and E. Knobloch, Phys. Rev. Lett. 95, 244501 (2005)

[6] O. Batiste et al., J. Fluid Mech. 560, 149 (2006).

[7] I. Mercader, A. Alonso, and O. Batiste, Phys. Rev. E 77, 036313 (2008).

[8] K. Ghorayeb and A. Mojtabi, Phys. Fluids 9, 2339 (1997); A. Bergeon and E. Knobloch, ibid. 20, 034102 (2008).
[9] S. Blanchflower, Phys. Lett. A 261, 74 (1999); J. H. P. Dawes, J. Fluid Mech. 570, 385 (2007).

[10] P. Assemat, A. Bergeon, and E. Knobloch, Fluid Dyn. Res. 40, 852 (2008).

[11] Y. Pomeau, Physica D 23, 3 (1986).

[12] A. Bergeon, J. Burke, E. Knobloch, and I. Mercader, Phys. Rev. E 78, 046201 (2008).

[13] J. Burke and E. Knobloch, Phys. Rev. E 73, 056211 (2006); G. Kozyreff and S. J. Chapman, Phys. Rev. Lett. 97, 044502 (2006); S. J. Chapman and G. Kozyreff, Physica D 238, 319 (2009).

[14] J. Burke and E. Knobloch, Phys. Lett. A 360, 681 (2007); Chaos 17, 037102 (2007).

[15] J. H. P. Dawes (unpublished).

[16] S. M. Houghton and E. Knobloch, Phys. Rev. E 80, 026210 (2009). 